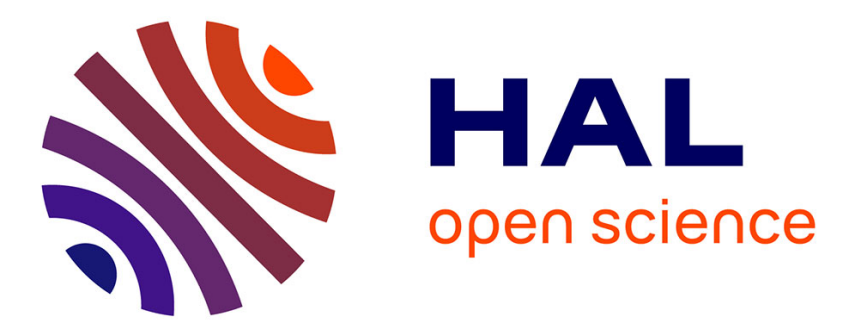

\title{
Interatomic potentials for alloys: Fitting concentration dependent properties
}

\author{
Giovanni Bonny, Roberto C Pasianot, Lorenzo Malerba
}

\section{To cite this version:}

Giovanni Bonny, Roberto C Pasianot, Lorenzo Malerba. Interatomic potentials for alloys: Fitting concentration dependent properties. Philosophical Magazine, 2009, 89 (08), pp.711-725. 10.1080/14786430902720994. hal-00514012

\section{HAL Id: hal-00514012 \\ https://hal.science/hal-00514012}

Submitted on 1 Sep 2010

HAL is a multi-disciplinary open access archive for the deposit and dissemination of scientific research documents, whether they are published or not. The documents may come from teaching and research institutions in France or abroad, or from public or private research centers.
L'archive ouverte pluridisciplinaire HAL, est destinée au dépôt et à la diffusion de documents scientifiques de niveau recherche, publiés ou non, émanant des établissements d'enseignement et de recherche français ou étrangers, des laboratoires publics ou privés. 


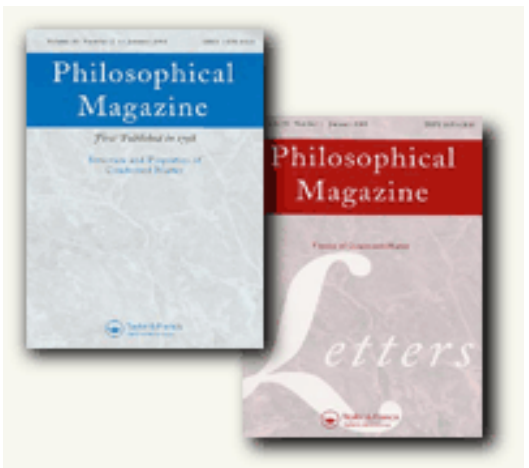

\section{Interatomic potentials for alloys: Fitting concentration dependent properties}

\begin{tabular}{|r|l|}
\hline Journal: & Philosophical Magazine \& Philosophical Magazine Letters \\
\hline Manuscript ID: & TPHM-08-Oct-0355.R1 \\
\hline Journal Selection: & Philosophical Magazine \\
\hline Author: & 29-Dec-2008 \\
\hline Komplete List of Authors: & $\begin{array}{l}\text { Bonny, Giovanni; SCK-CEN, Nuclear Materials Science Institute } \\
\text { Pasianot, Roberto; CAC/CNEA, Deparamento de Materiales } \\
\text { Malerba, Lorenzo; SCK-CEN, Nuclear Materials Science Institute }\end{array}$ \\
\hline Keywords (user supplied): & $\begin{array}{l}\text { Embedded atom method, Two-band model, Concentration } \\
\text { dependent method }\end{array}$ \\
\hline & \\
\hline $\begin{array}{l}\text { Note: The following files were submitted by the author for peer review, but cannot be converted } \\
\text { to PDF. You must view these files (e.g. movies) online. }\end{array}$ \\
\hline \begin{tabular}{l} 
PaperRevised.tex \\
\hline
\end{tabular}
\end{tabular}

\section{S ScholaroNE \\ Manuscript Central}


Philosophical Magazine

Vol. 00, No. 00, 00 Month 200x, 1-15

\title{
RESEARCH ARTICLE
}

\section{Interatomic potentials for alloys: Fitting concentration dependent properties}

\author{
G. Bonny ${ }^{\mathrm{a}, \mathrm{b} *}$, R.C. Pasianot ${ }^{\mathrm{c}, \mathrm{d}, \mathrm{e}}$ and L. Malerba ${ }^{\mathrm{a}}$

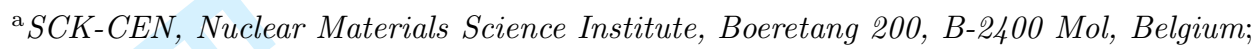 \\ ${ }^{\mathrm{b}}$ Center for Molecular Modeling, Ghent University, Proeftuinstraat 86, B-9000 Gent, \\ Belgium; ${ }^{\circ}$ CAC-CNEA, Departamento de Materiales, Avda. Gral. Paz 1499, 1650 San \\ Martín, Pcia. Buenos Aires, Argentina; ${ }^{\mathrm{d}}$ CONICET; ${ }^{\mathrm{e}}$ Instituto Sábato, UNSAM/CNEA
}

(Received 00 Month 200x; final version received 00 Month 200x)

\begin{abstract}
A detailed analysis of the embedded atom method and Finnis-Sinclair formalisms is performed, showing their limitations to fit concentration dependent properties of alloys. Two empirical extensions of those, so called two-band model and concentration dependent model, are analysed in depth, and their heuristic equivalence is shown. An algorithm is proposed for the former extension, capable of fitting concentration dependent properties of the alloy, such as mixing enthalpy and bulk modulus. The algorithm is then applied to the Fe-Cr system, deriving two interatomic potentials that closely reproduce Fe-Cr's complex mixing enthalpy.
\end{abstract}

Keywords: Interatomic potential; Embedded atom method; Finnis-Sinclair; Two-band model; Concentration dependent method; disordered systems

\section{Introduction}

In the past decades, computer simulations at the atomic level have become increasingly important, complementing experiments in materials science. Massive simulations, such as molecular dynamics and Monte Carlo, containing millions of atoms are now possible thanks to interatomic potentials which allow fast evaluation of the interactions in an empirical way. Besides experiment, fitting interatomic potentials to density functional theory (DFT) data has nowadays become a common practice. This methodology allows access, in a reliable way, to physical quantities that are experimentally impossible or too difficult to obtain. An example is the mixing enthalpy of Fe-Cr alloys, that changes sign at about 4 at.\% $\mathrm{Cr}$ [1-5]. For this particular system, trial and error has shown that traditional many-body potentials formalisms, such as the embedded atom method (EAM) [6] and FinnisSinclair (FS) [7], are not adequate to reproduce the complex shape of the mixing enthalpy and its implications on the alloy, e.g. short range ordering [8, 9]. Two empirical extensions of those were developed independently for the $\mathrm{Fe}-\mathrm{Cr}$ system to cope with this situation, namely, the two-band model (2BM)[10], and the concentration dependent model (CDM) [11]. The 2BM accounts for contributions from s-band electrons (besides the d-band electrons in FS) in the energy, as introduced by Ackland and Reed [12]. The CDM on the other hand follows a more empirical

*Corresponding author. Email: gbonny@sckcen.be

ISSN: 1478-6435 print/ISSN 1478-6443 online

(C) 200x Taylor \& Francis

DOI: $10.1080 / 1478643 Y Y x x x x x x x x$

http://www.informaworld.com 
path and introduces a local concentration dependence akin to the Redlich-Kister expansion [13] of the CALPHAD methodology [14].

In this work a detailed analysis is presented (section 2), proving that both EAM and FS are indeed not suitable to reproduce complex functions of concentration, and that $2 \mathrm{BM}$ and $\mathrm{CDM}$ are both equally suited to describe concentration dependencies in alloys. Based on this analysis, a heuristic equivalence between 2BM and $\mathrm{CDM}$ is shown, and illustrated by an example for the Fe-Cr alloy. Reversing the problem, the analytical formulae derived here are used for the development of a fitting algorithm (section 3), capable of matching very closely the concentration dependence of the average site energy and its derivatives, e.g. mixing enthalpy and elastic constants. The algorithm is then applied to fit 2BM potentials (section 4), that reproduce the mixing enthalpy behaviour of Fe-Cr alloys.

\section{The energy of random alloys}

The work of Ackland and Vitek [15] was among the first to consider the energetics of random alloys in the context of developing many-body FS potentials. All the fcc binaries built upon $\mathrm{Ag}, \mathrm{Au}$, and $\mathrm{Cu}$ were tackled, acknowledging the full statistical nature of the problem in the species concentration. An important outcome of their analysis was that when the lattice parameter mismatch is relatively large $(\mathrm{Cu}-$ $\mathrm{Ag}$ and $\mathrm{Cu}-\mathrm{Au}$ ), the local atomic relaxations make a sizable contribution to the energy, thus complicating the fitting. Our current approach is simpler, because local relaxations are neglected (this not being a restrictive hypothesis for the systems in mind, e.g. Fe-Cr). It is also more detailed, in the sense that the statistics is analytically carried out up to variance terms, thereby providing explicit and fairly reliable expressions upon which the energetic analysis is based.

\subsection{Average site energy}

The starting point is the concentration weighted combination of the average site energy for species A and B,

$$
\bar{E}(x)=x_{\mathrm{A}} \bar{E}_{\mathrm{A}}(x)+x_{\mathrm{B}} \bar{E}_{\mathrm{B}}(x),
$$

where $x_{\mathrm{A}}, x_{\mathrm{B}}$ denote the atomic fractions so that $x_{\mathrm{A}}+x_{\mathrm{B}}=1$, and $x$ stands for any one of them. Assuming the atoms are distributed in a purely random manner, the probability to find exactly $n_{\mathrm{A}}^{\nu} / n_{\mathrm{B}}^{\nu}$ atoms of type A / B in shell $\nu$, is given by the binomial distribution,

$$
P\left(n_{\mathrm{A}}^{\nu}, n_{\mathrm{B}}^{\nu}\right)=\frac{Z_{\nu} !}{n_{\mathrm{A}}^{\nu} ! n_{\mathrm{B}}^{\nu} !} x^{n_{\mathrm{A}}^{\nu}} x^{n_{\mathrm{B}}^{\nu}},
$$

where $Z_{\nu}=n_{\mathrm{A}}^{\nu}+n_{\mathrm{B}}^{\nu}$ denotes the total number of atoms in the shell. Correspondingly, the average and variance of e.g. $n_{\mathrm{A}}^{\nu}$ are,

$$
\begin{gathered}
\overline{n_{\mathrm{A}}^{\nu}}=Z_{\nu} x_{\mathrm{A}} \\
\overline{\left(n_{\mathrm{A}}^{\nu}-\overline{n_{\mathrm{A}}^{\nu}}\right)^{2}}=Z_{\nu} x_{\mathrm{A}} x_{\mathrm{B}},
\end{gathered}
$$

results extensively used below, together with the hypothesis of statistical independence among shells. 
Within EAM or FS the site energy $E_{\mathrm{A}}$ (symmetric for $E_{\mathrm{B}}$ ) is given as,

$$
E_{\mathrm{A}}=\frac{1}{2} \sum_{\nu} n_{\mathrm{A}}^{\nu} V_{\mathrm{AA}}^{\nu}+\frac{1}{2} \sum_{\nu} n_{\mathrm{B}}^{\nu} V_{\mathrm{AB}}^{\nu}+F_{\mathrm{A}}\left(\rho_{\mathrm{A}}\right)
$$

where $V$ are pair terms, $F$ is the embedding term and $\rho$ an heuristic electron density (EAM) or second moment of the d-electron band (FS); besides, $\nu$ runs over all the nearest neighbour shells within the cut-off range of the potential, and $V^{\nu}=V\left(r_{\nu}\right)$ with $r_{\nu}$ the shell radius. In the FS formalism $F$ explicitly scales as $\sqrt{\rho}$. In order to obtain the average site energy $\bar{E}_{\mathrm{A}}$, equation (3) is applied to equation (5). The contribution from the pair terms is trivial; the one from the embedding terms, being the electron densities $\rho_{\mathrm{A}}$ and $\rho_{\mathrm{B}}$ derived statistical variables, requires further treatment. Assuming that $F_{\mathrm{A}}$ and $F_{\mathrm{B}}$ are Taylor expandable about the average electron densities $\bar{\rho}_{\mathrm{A}}$ and $\bar{\rho}_{\mathrm{B}}$, we obtain (up to second order),

$$
F_{\mathrm{A}}\left(\rho_{\mathrm{A}}\right)=F_{\mathrm{A}}\left(\bar{\rho}_{\mathrm{A}}\right)+\left(\rho_{\mathrm{A}}-\bar{\rho}_{\mathrm{A}}\right) F_{\mathrm{A}}^{\prime}\left(\bar{\rho}_{\mathrm{A}}\right)+\frac{1}{2}\left(\rho_{\mathrm{A}}-\bar{\rho}_{\mathrm{A}}\right)^{2} F_{\mathrm{A}}^{\prime \prime}\left(\bar{\rho}_{\mathrm{A}}\right) .
$$

Thus the site average of the embedding terms $\overline{F_{\mathrm{A}}\left(\rho_{\mathrm{A}}\right)}$ can be written as,

$$
\overline{F_{\mathrm{A}}\left(\rho_{\mathrm{A}}\right)} \approx F_{\mathrm{A}}\left(\bar{\rho}_{\mathrm{A}}\right)+\frac{1}{2} \overline{\left(\rho_{\mathrm{A}}-\bar{\rho}_{\mathrm{A}}\right)^{2}} F_{\mathrm{A}}^{\prime \prime}\left(\bar{\rho}_{\mathrm{A}}\right) .
$$

For obvious reasons we call this approach the variance expansion. In turn, the density on a site occupied by A (analogue for B) is given by,

$$
\rho_{\mathrm{A}}=\sum_{\nu}\left(n_{\mathrm{A}}^{\nu} \varphi_{\mathrm{AA}}^{\nu}+n_{\mathrm{B}}^{\nu} \varphi_{\mathrm{AB}}^{\nu}\right)
$$

where $\varphi^{\nu}$ represents the density function evaluated at $\nu$-th shell. Again, after applying equations (3) and (4), one obtains,

$$
\bar{\rho}_{\mathrm{A}}(x)=\sum_{\nu} Z_{\nu}\left(x_{\mathrm{A}} \varphi_{\mathrm{AA}}^{\nu}+x_{\mathrm{B}} \varphi_{\mathrm{AB}}^{\nu}\right) ;
$$

and

$$
\overline{\left(\rho_{\mathrm{A}}-\bar{\rho}_{\mathrm{A}}\right)^{2}}(x)=x_{\mathrm{A}} x_{\mathrm{B}} \sum_{\nu} Z_{\nu}\left(\varphi_{\mathrm{AB}}^{\nu}-\varphi_{\mathrm{AA}}^{\nu}\right)^{2} .
$$

In the EAM formalism we choose $\varphi_{\mathrm{AA}}=\varphi_{\mathrm{BA}}=\varphi_{\mathrm{A}}$ and $\varphi_{\mathrm{BB}}=\varphi_{\mathrm{AB}}=\varphi_{\mathrm{B}}$, which simplifies the previous expressions so that $\bar{\rho}_{\mathrm{A}}=\bar{\rho}_{\mathrm{B}}$ and $\overline{\left(\rho_{\mathrm{A}}-\bar{\rho}_{\mathrm{A}}\right)^{2}}=\overline{\left(\rho_{\mathrm{B}}-\bar{\rho}_{\mathrm{B}}\right)^{2}}$.

The final expression for the average site energy within EAM or FS is therefore,

$$
\begin{aligned}
\bar{E}_{\mathrm{EAM}}(x) & =\sum_{\nu} \frac{Z_{\nu}}{2}\left[x_{\mathrm{A}}^{2} V_{\mathrm{AA}}^{\nu}+x_{\mathrm{B}}^{2} V_{\mathrm{BB}}^{\nu}+2 x_{\mathrm{A}} x_{\mathrm{B}} V_{\mathrm{AB}}^{\nu}\right] \\
& +x_{\mathrm{A}}\left[F_{\mathrm{A}}\left(\bar{\rho}_{\mathrm{A}}\right)+\frac{1}{2} \overline{\left(\rho_{\mathrm{A}}-\bar{\rho}_{\mathrm{A}}\right)^{2}} F_{\mathrm{A}}^{\prime \prime}\left(\bar{\rho}_{\mathrm{A}}\right)\right] \\
& +x_{\mathrm{B}}\left[F_{\mathrm{B}}\left(\bar{\rho}_{\mathrm{B}}\right)+\frac{1}{2} \overline{\left(\rho_{\mathrm{B}}-\bar{\rho}_{\mathrm{B}}\right)^{2}} F_{\mathrm{B}}^{\prime \prime}\left(\bar{\rho}_{\mathrm{B}}\right)\right],
\end{aligned}
$$

which is an analytic (though not explicit) function of the global concentration. 
In the $2 \mathrm{BM}$ formalism the site energy is augmented by the 's-embedding' terms, $F_{\mathrm{A}}^{\mathrm{s}}\left(\rho_{\mathrm{A}}^{\mathrm{s}}\right)$ and $F_{\mathrm{B}}^{\mathrm{s}}\left(\rho_{\mathrm{B}}^{\mathrm{s}}\right)$, respectively. The s-densities are now $\varphi_{\mathrm{AA}}^{\mathrm{s}}=\varphi_{\mathrm{BB}}^{\mathrm{s}}=0$ and $\varphi_{\mathrm{AB}}^{\mathrm{s}}=\varphi_{\mathrm{BA}}^{\mathrm{s}}$, so simplifying the average and variance as defined by equations (9) and (10). Thus, the average site energy becomes

$$
\begin{aligned}
\bar{E}_{2 \mathrm{BM}}(x) & =\bar{E}_{\mathrm{EAM}}(x)+x_{\mathrm{A}}\left[F_{\mathrm{A}}^{\mathrm{s}}\left(\bar{\rho}_{\mathrm{A}}^{\mathrm{s}}\right)+\frac{1}{2} \overline{\left(\rho_{\mathrm{A}}^{\mathrm{s}}-\bar{\rho}_{\mathrm{A}}^{\mathrm{s}}\right)^{2}} F_{\mathrm{A}}^{\mathrm{s}}{ }^{\prime \prime}\left(\bar{\rho}_{\mathrm{A}}^{\mathrm{s}}\right)\right] \\
& +x_{\mathrm{B}}\left[F_{\mathrm{B}}^{\mathrm{s}}\left(\bar{\rho}_{\mathrm{B}}^{\mathrm{s}}\right)+\frac{1}{2} \overline{\left(\rho_{\mathrm{B}}^{\mathrm{s}}-\bar{\rho}_{\mathrm{B}}^{\mathrm{s}}\right)^{2}} F_{\mathrm{B}}^{\mathrm{s}}{ }^{\prime \prime}\left(\bar{\rho}_{\mathrm{B}}^{\mathrm{s}}\right)\right]
\end{aligned}
$$

which is also an analytic function of the global concentration. Worth of notice is that, for the s-embedding function, the scaling $F^{\mathrm{s}} \propto \sqrt{\rho^{\mathrm{s}}}$ proposed in [10] is a rather problematic choice, since both $\rho^{\mathrm{s}}$ and $\overline{\rho^{\mathrm{s}}}$ approach zero in the dilute limits of the alloy, leading to singularities in the interatomic forces.

The CDM formalism is similar to EAM with the difference that the cross pair interaction includes a multiplicative factor, $h\left(x_{\mathrm{AB}}\right)$, which depends on a local concentration parameter $x_{\mathrm{AB}}$. With $x_{\mathrm{AB}}^{\nu} \equiv x_{\mathrm{AB}}\left(r_{\nu}\right)$, the average site energy is the same as equation (11), except for the cross pair contribution, that now reads,

$$
\sum_{\nu} Z_{\nu} V_{\mathrm{AB}}^{\nu} \rightarrow \sum_{\nu} Z_{\nu}\left(h\left(\bar{x}_{\mathrm{AB}}^{\nu}\right)+\frac{1}{2} \overline{\left(x_{\mathrm{AB}}^{\nu}-\bar{x}_{\mathrm{AB}}^{\nu}\right)^{2}} h^{\prime \prime}\left(\bar{x}_{\mathrm{AB}}^{\nu}\right)\right) V_{\mathrm{AB}}^{\nu}
$$

where a variance expansion of $\overline{h\left(x_{\mathrm{AB}}^{\nu}\right)}$ about the average $\bar{x}_{\mathrm{AB}}^{\nu}$ is used. The derivation of the above average and variance is somewhat more involved, though does not present any essential difficulty; it is given in appendix A.

\subsection{Energy of mixing}

The mixing enthalpy is defined as the difference between the average site energy and the energy of the mechanical mixture of the pure elements,

$$
E_{\text {mix }}(x)=\bar{E}(x)-x_{\mathrm{A}} E_{\mathrm{A}}-x_{\mathrm{B}} E_{\mathrm{B}} .
$$

Because of its relevance for the Fe-Cr binary alloy, it is of particular interest to us to develop the case where pure elements and alloy possess coherent structures, presently bcc. At a certain concentration the equilibrium lattice parameter is assumed to be $a(x)$; in terms of the pure elements lattice parameters we write (same for B),

$$
a(x)=a_{A}+\delta a_{A} \text { and } r_{\nu}(x)=r_{\nu}^{A}+\delta r_{\nu}^{A} .
$$

Assuming the changes are small, the pair potentials are expanded to first order as,

$$
V_{\mathrm{AA}}\left(r_{\nu}\right) \approx V_{\mathrm{AA}}\left(r_{\nu}^{\mathrm{A}}\right)+\delta r_{\nu}^{\mathrm{A}} V_{\mathrm{AA}}^{\prime}\left(r_{\nu}^{\mathrm{A}}\right)
$$

Thus we get for the pair contribution to the mixing enthalpy,

$$
E_{\mathrm{mix}}^{\mathrm{pair}}(x)=x_{\mathrm{A}} x_{\mathrm{B}} \sum_{\nu} \frac{Z_{\nu}}{2}\left(2 V_{\mathrm{AB}}^{\nu}-V_{\mathrm{AA}}^{\nu}-V_{\mathrm{BB}}^{\nu}\right)+\delta V .
$$


where $\delta V$ is a residual term, linear in the lattice parameter deviations,

$$
\delta V=-x_{\mathrm{A}} \sum_{\nu} \frac{Z_{\nu}}{2} \delta r_{\nu}^{\mathrm{A}} V_{\mathrm{AA}}^{\prime}(\nu)-x_{\mathrm{B}} \sum_{\nu} \frac{Z_{\nu}}{2} \delta r_{\nu}^{B} V_{\mathrm{BB}}^{\prime}(\nu)
$$

If $a(x)$ is a linear function (simplest case), $\delta V$ is quadratic, making the term symmetric about $x=0.5$. As a consequence, when taking $V_{\mathrm{AB}}$ as the average of the pure species (as in CDM), $E_{\text {mix }}^{\text {pair }}(x)$ can only reproduce symmetric parabolic forms. Any other shape is due to non-linearities in the $a(x)$ relationship.

Regarding the embedding terms contribution, from equation (11) one obtains,

$$
\begin{aligned}
E_{\mathrm{mix}}^{\mathrm{emb}}(x) & =x_{\mathrm{A}}\left(F_{\mathrm{A}}\left(\bar{\rho}_{\mathrm{A}}\right)-F_{\mathrm{A}}\left(\rho_{\mathrm{A}}^{0}\right)+\frac{1}{2} x_{\mathrm{A}} x_{\mathrm{B}} \overline{\left(\rho_{\mathrm{A}}-\bar{\rho}_{\mathrm{A}}\right)^{2}} F_{\mathrm{A}}^{\prime \prime}\left(\bar{\rho}_{\mathrm{A}}\right)\right) \\
& +x_{\mathrm{B}}\left(F_{\mathrm{B}}\left(\bar{\rho}_{\mathrm{B}}\right)-F_{\mathrm{B}}\left(\rho_{\mathrm{B}}^{0}\right)+\frac{1}{2} x_{\mathrm{B}} x_{\mathrm{A}} \overline{\left(\rho_{\mathrm{B}}-\bar{\rho}_{\mathrm{B}}\right)^{2}} F_{\mathrm{B}}^{\prime \prime}\left(\bar{\rho}_{\mathrm{B}}\right)\right)
\end{aligned}
$$

where $\rho_{\mathrm{A}}^{0}\left(\rho_{\mathrm{B}}^{0}\right)$ is the equilibrium density in pure $\mathrm{A}(\mathrm{B})$. For the pure elements, the density units are essentially arbitrary: rescaling the density simply entails a redefinition of the embedding term in order to obtain the same energy per atom [7]. In the alloy, however, the density on an $\mathrm{A}$ atom is contributed also by $\mathrm{B}$ atoms and conversely, which means that the strength of the B contribution as seen by the A species must be decided. Within the image of rigid electron clouds, a convenient way to express this is by fixing the ratio $\rho_{\mathrm{B}} / \rho_{\mathrm{A}}=\chi$ for some common lattice. In what follows and to avoid non-essential complications, the somewhat stronger assumption $\varphi_{\mathrm{B}}(r) / \varphi_{\mathrm{A}}(r)=\chi$ is made, being $\chi$ a number of order unity. Accordingly, we write,

$$
\rho_{\mathrm{B}}^{0}=\rho_{\mathrm{A}}^{0}+\delta \rho^{0} \text { with } \delta \rho^{0} \approx(\chi-1) \rho_{\mathrm{A}}^{0} .
$$

On the other hand, for the average densities,

$$
\bar{\rho}_{\mathrm{A}}=\rho_{\mathrm{A}}^{0}+\delta \rho_{\mathrm{A}} \text { and } \bar{\rho}_{\mathrm{B}}=\rho_{\mathrm{B}}^{0}+\delta \rho_{\mathrm{B}},
$$

where $\delta \rho_{\mathrm{A}}$ (symmetric for $\delta \rho_{\mathrm{B}}$ ) is expressed by,

$$
\delta \rho_{\mathrm{A}}=x_{\mathrm{B}} \delta \rho^{0}-\sum_{\nu} Z_{\nu}\left[x_{\mathrm{A}} \delta r_{\nu}^{\mathrm{A}} \varphi_{\mathrm{A}}^{\prime}\left(r_{\nu}^{\mathrm{A}}\right)+x_{\mathrm{B}} \delta r_{\nu}^{\mathrm{B}} \varphi_{\mathrm{B}}^{\prime}\left(r_{\nu}^{\mathrm{B}}\right)\right]
$$

that also includes the contribution due to the small deviations in the lattice parameter with respect to the pure species ones. If $\chi=1$ the latter is the only contribution, and we are back to a similar analysis than for pure pair interactions. In fact, because we want to analyze effects inherent to the EAM form, the point of view is rather the opposite: assume $\chi \neq 1$ and basically neglect the terms from lattice parameter mismatch. Continuing with the analysis of equation (19), the embedding functions are now expanded as,

$$
F_{\mathrm{A}}\left(\bar{\rho}_{\mathrm{A}}\right)=F_{\mathrm{A}}\left(\rho_{\mathrm{A}}^{0}\right)+\delta \rho_{\mathrm{A}} F_{\mathrm{A}}^{\prime}\left(\rho_{\mathrm{A}}^{0}\right)+\frac{1}{2}\left(\delta \rho_{\mathrm{A}}\right)^{2} F_{\mathrm{A}}^{\prime \prime}\left(\rho_{\mathrm{A}}^{0}\right),
$$

where, without loss of generality, one may set $F_{\mathrm{A}}^{\prime}\left(\rho_{\mathrm{A}}^{0}\right)=0$ and $F_{\mathrm{B}}^{\prime}\left(\rho_{\mathrm{B}}^{0}\right)=0$, according to the effective potential gauge for the pure elements [7]. We further 
define,

$$
\varphi_{\mathrm{B}}^{\nu}=\varphi_{\mathrm{A}}^{\nu}+\delta \varphi_{\nu} \text { with } \delta \varphi_{\nu}=(\chi-1) \varphi_{\mathrm{A}}^{\nu}
$$

that leads to the variance factors,

$$
\begin{aligned}
\overline{\left(\rho_{\mathrm{A}}-\bar{\rho}_{\mathrm{A}}\right)^{2}} & =(\chi-1)^{2} x_{\mathrm{A}} x_{\mathrm{B}} \sum_{\nu} Z_{\nu}\left(\varphi_{\mathrm{A}}^{\nu}\right)^{2} \\
& =\overline{\left(\rho_{\mathrm{B}}-\bar{\rho}_{\mathrm{B}}\right)^{2}}
\end{aligned}
$$

so, gathering these results in our starting formula,

$$
\begin{aligned}
E_{\text {mix }}^{\mathrm{emb}}(x) & \approx(\chi-1)^{2}\left\{\left(x_{\mathrm{A}} F_{\mathrm{A}}^{\prime \prime}\left(\rho_{\mathrm{A}}^{0}\right)+x_{\mathrm{B}} F_{\mathrm{B}}^{\prime \prime}\left(\rho_{\mathrm{B}}^{0}\right)\right) Q_{4}(x)\right. \\
& \left.+\left(x_{\mathrm{A}} F_{\mathrm{A}}^{\prime \prime}\left(\bar{\rho}_{\mathrm{A}}\right)+x_{\mathrm{B}} F_{\mathrm{B}}^{\prime \prime}\left(\bar{\rho}_{\mathrm{B}}\right)\right) Q_{2}(x)\right\}
\end{aligned}
$$

with,

$$
\begin{aligned}
& Q_{4}(x) \equiv \frac{1}{2} x_{\mathrm{A}}^{2} x_{\mathrm{B}}^{2} \sum_{\nu}\left(\varphi_{\mathrm{A}}^{\nu}\right)^{2} \\
& Q_{2}(x) \equiv \frac{1}{2} x_{\mathrm{A}} x_{\mathrm{B}}\left(\delta \rho^{0}\right)^{2}
\end{aligned}
$$

A number of conclusions can be drawn from the above. First, an asymmetric mixing enthalpy curve is possible only if $F_{\mathrm{A}}^{\prime \prime} \neq F_{\mathrm{B}}^{\prime \prime}$. However, in our implicit assumption that the pure element potentials are given and that we only work on the cross potential, these are not parameters at our disposal (e.g. for the cubic structure $F^{\prime \prime}$ is essentially fixed by the Cauchy pressure $\left.\left(C_{12}-C_{44}\right)\right)$. Second, the only parameter at our disposal to connect to alloy data is a scale change $(\chi-1)^{2}$, particularly the zeros of equation (26) are fixed beforehand, all of which represents a severe lack of flexibility for the purpose of fitting. For instance, it is easy to see that the above cannot sustain a shape like in $\mathrm{Fe}-\mathrm{Cr}$, where the mixing enthalpy fulfills $H_{\text {mix }}\left(x_{1}\right)=0$ for some $x_{1}$ close to 0 (now $x \equiv x_{\mathrm{B}} \equiv x_{\mathrm{Cr}}$ ), being negative for $x \in\left[0, x_{1}\right]$. In the vicinity $x \sim 0$ one may supplement equation (26) with a pair contribution like equation (17) and write

$$
H_{\text {mix }} \approx x(1-x)\left[F_{\mathrm{B}}^{\prime \prime}\left(\delta \rho^{0}\right)^{2}(1-x) / 2-V\right],
$$

where the constant $V$ complies with $V>F_{\mathrm{B}}^{\prime \prime}\left(\delta \rho^{0}\right)^{2} / 2$; clearly this function does not turn upwards fast enough to approach the zero at $x_{1}$. Thus, this analysis shows that the EAM formalism is unsuited to fit mixing properties with complexity greater than a third order polynomial in $x$.

In the 2BM scheme, the s-embedding terms of equation (12) contribute fully to the mixing enthalpy. The average s-densities scale linearly with concentration and therefore the local s-density also estimates the local concentration at a certain atomic site. In fact, the above is similar to the EAM form, equation (19), the difference now being that the s-embeddings are functions to be determined (without effect on the pure species), so that the order of complexity of $F_{\mathrm{A}}^{\mathrm{s}}$ and $F_{\mathrm{B}}^{\mathrm{s}}$ allows for quite general shapes for the mixing enthalpy. In principle, by choosing a high order polynomial, or a spline expansion with enough knots, any mixing enthalpy shape can be reproduced. The fitting itself, however, may not be straightforward. 
Within the CDM formalism, the mixing enthalpy can be expanded as,

$$
E_{\text {mix }}^{\mathrm{cdm}}(x)=E_{\text {mix }}^{\mathrm{eam}}(x)+\epsilon_{\mathrm{mix}}^{\mathrm{cdm}}(x)
$$

where $E_{\text {mix }}^{\mathrm{eam}}(x)=E_{\mathrm{mix}}^{\mathrm{pair}}(x)+E_{\mathrm{mix}}^{\mathrm{emb}}(x)$, and (c.f. appendix A)

$$
\begin{aligned}
\epsilon_{\text {mix }}^{\mathrm{cdm}}(x) & \approx x_{\mathrm{A}} x_{\mathrm{B}} \sum_{\nu} Z_{\nu} V_{\mathrm{AB}}^{\nu} \\
& \times\left(h(x)+C x_{\mathrm{A}} x_{\mathrm{B}} \frac{\sum_{\nu} Z_{\nu}\left(\varphi^{\nu}\right)^{2}}{2 \rho^{2}} h^{\prime \prime}(x)-1\right)
\end{aligned}
$$

with $C \in[0.5,1]$. Thus the order of complexity of the modulation factor $h(x)$ determines the possible shapes of mixing enthalpy curves. In summary, both the $2 \mathrm{BM}$ and CDM offer in principle adequate frameworks to fit concentration dependent properties, while the EAM or FS lack flexibility for complex dependencies.

\section{Fitting algorithm}

From the analysis in the previous section it has become clear that the EAM formalism can reproduce mostly parabolic mixing enthalpy curves, and that non-negligible deviations from that shape may result from the variation of lattice parameter with concentration. On the other hand it has been shown that both CDM and 2BM can reproduce any shape of the mixing enthalpy. This because, in both formalisms, the EAM was supplemented by an energy term dependent on a 'local concentration'. In appendix B it is shown that, although no one-to-one relationship exists between CDM and 2BM, both formalisms are equivalent in describing concentration dependences. Being this the case, in the following we restrict ourselves to fitting algorithms optimized for 2BM.

Even though the possibility of an exact fit (of the average site energy and its derivatives) has been shown, the fitting problem itself is not straightforward. The challenge is that, in the expression for the mixing enthalpy, the equilibrium lattice parameter as a function of concentration is needed, which in turn is determined by the potential to be fitted. This makes the objective function of the fit highly non-linear in its fitting parameters.

Before fitting, both pure potentials (taken from the literature) are transformed to the effective gauge form [7], so that $F^{\prime}\left(\rho_{\text {eq }}\right)=0$ and $\rho_{\text {eq }}=1$ at their equilibrium lattices. Note this does not affect the properties of the pure species. In the fitting itself, all efforts are focused on the cross pair potential and s-embedding functions, while the s-density function is a priori fixed. Then, our procedure runs as follows:

(i) Given an appropriate s-density function $\varphi_{\mathrm{AB}}^{\mathrm{s}}$, choose a cross pair potential $V_{\mathrm{AB}}$ and lattice parameter relationship $a(x)$.

(ii) Fit the s-embedding terms $F_{\mathrm{A}}^{\mathrm{s}}$ and $F_{\mathrm{B}}^{\mathrm{s}}$; this involves the solution of a set of two differential equations or, when choosing a spline form, the solving of a quadratic programming problem.

(iii) Keep the s-embedding terms $F_{\mathrm{A}}^{\mathrm{s}}$ and $F_{\mathrm{B}}^{\mathrm{s}}$ obtained in the previous step fixed, and re-fit the cross pair potential $V_{\mathrm{AB}}$ with the constraints 1 ) that the contribution of the newly fitted cross pair potential to the mixing enthalpy must be the same as the old one, and 2) that the resulting equilibrium parameters at different concentrations match the curve $a(x)$ chosen in step (i) (zero pressure condition). The quality of the fit is dominated by how 
well these constraints are satisfied.

In step (i) the s-density function $\varphi_{\mathrm{AB}}^{\mathrm{s}}$ can be chosen as monotonically decreasing, similarly to the traditional EAM, or non-monotonic and, moreover, satisfying the condition,

$$
\left.\sum_{\nu} Z_{\nu} \frac{\partial \varphi_{\mathrm{AB}}^{\mathrm{s}}(r)}{\partial r}\right|_{r=r_{\nu}}=0
$$

which makes the s-embedding energy stationary with respect to small changes in the equilibrium lattice parameter, and reduces the zero pressure condition posed in step (iii) to the EAM form. Here the s-density functions are selected to be Gaussian,

$$
\begin{aligned}
& \varphi_{\mathrm{AB}}^{\mathrm{s}}(r)=C_{0} \exp \left(-\alpha r^{2}\right) \\
& \varphi_{\mathrm{AB}}^{\mathrm{s}}(r)=\left\{\begin{array}{l}
C_{0} \exp \left(-\alpha_{1}\left(r-r_{0}\right)^{2}\right), r \leq r_{0} \\
C_{0} \exp \left(-\alpha_{2}\left(r-r_{0}\right)^{2}\right), r>r_{0}
\end{array}\right.
\end{aligned}
$$

being the first a monotonic curve and the second a non-monotonic one forced to satisfy equation (32). In equation (33) the parameter $\alpha$ is chosen as a compromise to make the function as constant as possible, while still decreasing fast enough to ensure a smooth cut-off between fifth nearest neighbour (5nn) and 6nn. For the cross pair potential a linear combination of the pure species ones is chosen at the start, e.g., the arithmetic mean,

$$
V_{\mathrm{AB}}=\frac{1}{2}\left(V_{\mathrm{AA}}+V_{\mathrm{BB}}\right)
$$

Such a choice yields only minor violations of the zero pressure of the EAM part, since the condition is satisfied by both pure potentials. It is noted that for the alloy the zero pressure requirement can be satisfied exactly at only one concentration (even in the EAM case). For the lattice parameter, a curve fitted to experimental data can be chosen; here, however, for the sake of simplicity we take a linear interpolation between the pure species ones,

$$
a(x)=a_{\mathrm{A}}+x\left(a_{\mathrm{B}}-a_{\mathrm{A}}\right) .
$$

Since only one point in $x$ of $a(x)$ can be matched exactly, a trade-off with fitting the mixing enthalpy is necessary, we have thus emphasized the latter.

In step (ii) the s-embedding functions $F_{\mathrm{A}}^{\mathrm{s}}$ and $F_{\mathrm{B}}^{\mathrm{s}}$ were parameterized using quartic splines,

$$
F^{\mathrm{S}}(\rho)=\sum_{k=1}^{\mathrm{N}} f_{k}\left(\rho-\rho_{k}\right)^{4} H\left(\rho-\rho_{k}\right)
$$

where $f_{k}$ are the fitting coefficients, $\rho_{k}$ the matching knots, and $H(x)$ the Heaviside unit step. Within the assumptions made in step (i), the mixing enthalpy (as derived in the previous section) results a linear expression in the fitting coefficients of the quartic forms for $F_{\mathrm{A}}^{\mathrm{s}}$ and $F_{\mathrm{B}}^{\mathrm{s}}$. When using as target function the statistical variance between data and mixing enthalpy (a very common practice), one obtains a quadratic programming problem that is exactly solvable.

In step (iii) the s-embedding functions obtained from step (ii) are kept fixed and the cross pair potential $V_{\mathrm{AB}}$ is re-fitted. For $V_{\mathrm{AB}}$ a standard cubic spline expansion 
is chosen,

$$
V(r)=\sum_{k=1}^{\mathrm{N}} v_{k}\left(r_{k}-r\right)^{3} H\left(r_{k}-r\right)
$$

where $v_{k}$ are the fitting coefficients and $r_{k}$ the matching knots. Note that this expansion has a natural cut-off, namely, the largest $r_{k}$. The new cross pair potential is forced to reproduce the same mixing enthalpy contribution as the old one, a constraint that can be expressed as,

$$
\sum_{\nu} Z_{\nu} V_{\mathrm{AB}}^{\text {new }}\left(r_{\nu}\right)=\sum_{\nu} Z_{\nu} V_{\mathrm{AB}}^{\text {old }}\left(r_{\nu}\right)
$$

For a proper mixing enthalpy fit, the lattice parameter $a(x)$ must be the same, after relaxation, to the equilibrium value; this condition is expressed by the zero pressure requirement,

$$
\left.\frac{d H_{\text {mix }}(x)}{d a}\right|_{a=a(x)}=0, \quad \forall x .
$$

The above proves to be very difficult to satisfy for a monotonically decreasing sdensity, but is almost automatically satisfied using a non-monotonic one complying with equation (32). The latter is an attractive solution, but has the side effect that the forces due to the s-embedding term change sign within the cut-off range and we suspect that this might introduce unwanted effects in dynamic simulations. At this point $V_{\mathrm{AB}}$ can also be re-fitted using a non-linear optimization routine, to match other properties of the alloy as well.

As a general comment, the number of free parameters amount to the coefficients of the cross-pair potential and those of the embedding functions (c.f. Table 1). Generally, these are few in number compared to the restrictions imposed by the data to be fitted. In principle that number can be increased at will, however, experience shows that beyond a certain loose optimum, the quality of the fit does not necessarily improve, and clearly unphysical behaviour appears in the functions shape, such as arbitrary oscillations.

As a final remark, we note that the cross pair potential $V_{\mathrm{AB}}$ and the s-embedding terms $F_{\mathrm{A}}^{\mathrm{s}}$ and $F_{\mathrm{B}}^{\mathrm{s}}$ within the $2 \mathrm{BM}$ are not uniquely determined. There exist two types of symmetry transformations, $T_{1}$ and $T_{2}$, analogous to the ones valid within the EAM formalism for the pure species [7], that keep the total energy invariant. These transformations are,

$$
\begin{gathered}
T_{1}:\left\{\begin{array}{l}
\varphi_{\mathrm{AB}}^{\mathrm{s}}(r) \rightarrow S \varphi_{\mathrm{AB}}^{\mathrm{s}}(r) \\
F^{\mathrm{s}}(\rho) \rightarrow F^{\mathrm{s}}(\rho / S)
\end{array}\right. \\
T_{2}:\left\{\begin{array}{l}
F^{\mathrm{s}}(\rho) \rightarrow F^{\mathrm{s}}(\rho)+C \rho \\
V_{\mathrm{AB}}(r) \rightarrow V_{\mathrm{AB}}(r)-2 C \varphi_{\mathrm{AB}}^{\mathrm{s}}(r)
\end{array},\right.
\end{gathered}
$$

with $S$ and $C$ constants. 


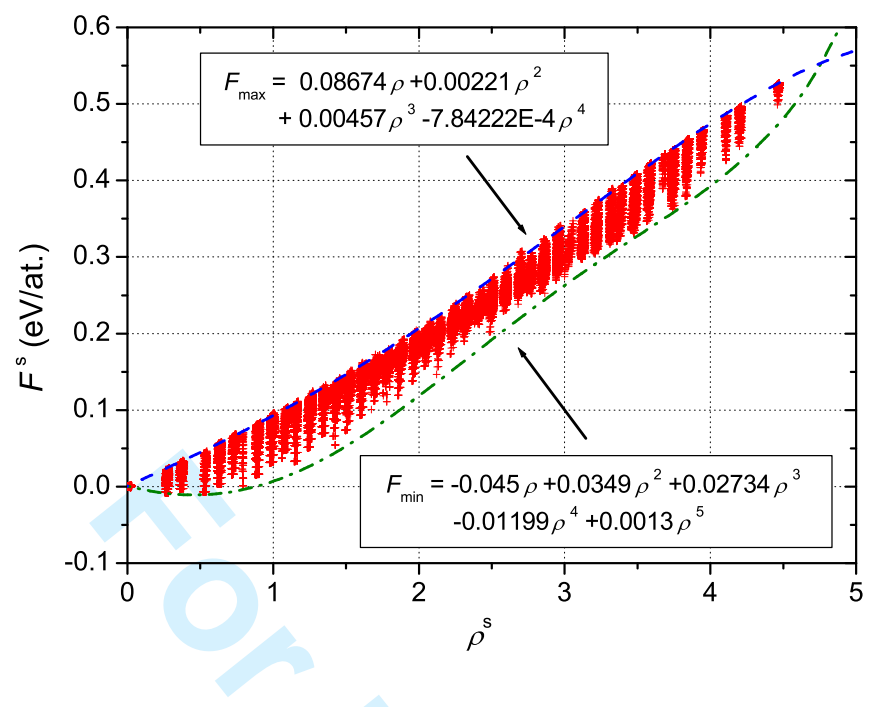

Figure 1. Possible s-embedding functions for Fe as a function of s-density.

\section{Applications}

In appendix B the equivalence in alloy energetics between 2BM and CDM has been suggested. However, no one-to-one relationship is possible between $\rho^{\mathrm{s}}$ and $x_{\mathrm{AB}}$, since both quantities depend on the local concentration, which can be different from the global concentration $x$. Therefore, it is not possible to make a 2BM potential which is an exact replica of a CDM potential. However, in terms of mixing enthalpy, equations (B5) and (B6) can be used to find a relationship between the potentials. As an example, the Fe-Cr CDM potential developed in [11] is converted into a 2BM potential. For simplicity, the s-embedding function $F_{\mathrm{B}}^{\mathrm{s}}$ is set to zero in the whole density range. Doing so, equation (B5) simplifies to

$$
F_{\mathrm{A}}^{\mathrm{s}}\left(\rho_{i}^{\mathrm{s}}\right)=\left(1-h\left(x_{i j}\right)\right) \rho_{i}^{\mathrm{s}} .
$$

Relationship (B6) defining the s-density function is easily implemented, but equation (43) introduces some difficulties. Since it is determined by local quantities, the relationship was sampled by calculating the local concentration dependent parameters $x_{i j}$ and $\rho_{i}^{\mathrm{s}}$ for several atomic configurations. The latter were taken from bcc simulation boxes containing 250 atoms of the disordered $\mathrm{Fe}_{1-x} \mathrm{Cr}_{x}$ alloy. In our computations every concentration $x$ was sampled with a 0.01 step interval. Thus, for a single global concentration, multiple values for the local variables were obtained, so that no one-to-one relationship between $F_{\mathrm{A}}^{\mathrm{s}}$ and $\rho^{\mathrm{s}}$ exists.

The result of this procedure is presented by the scatter in figure 1. From the figure two extreme s-embedding functions, $F_{\min }$ and $F_{\max }$, are derived. Identifying $\mathrm{A}$ with $\mathrm{Fe}$ and $\mathrm{B}$ with $\mathrm{Cr}$, in figure 2 the mixing enthalpies resulting from the two derived $2 \mathrm{BM}$ potentials are compared with the one from the CDM potential. The CDM curve is enclosed by the other two curves. It is thus clear that there exists at least one curve $F_{\mathrm{A}}^{\mathrm{s}}$ between the extremes $F_{\min }$ and $F_{\max }$ that exactly reproduces the CDM result. In summary, the example clearly shows the equivalence in alloy energetics between the two models.

As an application of the fitting algorithm presented in section 3, two 2BM potentials have been fitted to the most recent DFT calculated mixing enthalpy for 


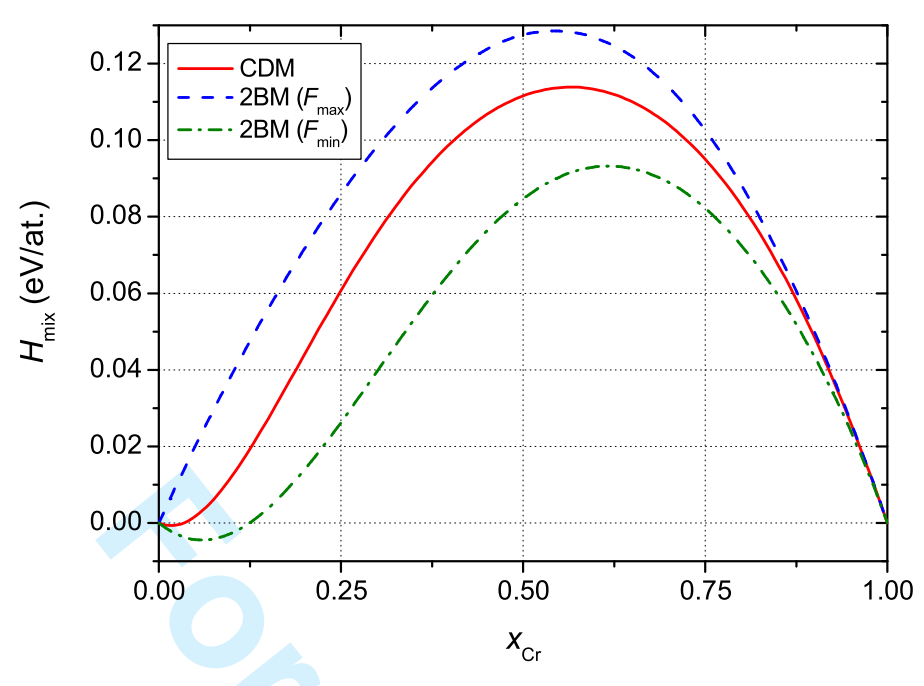

Figure 2. Mixing enthalpy for the CDM and therefrom derived 2BM potentials.

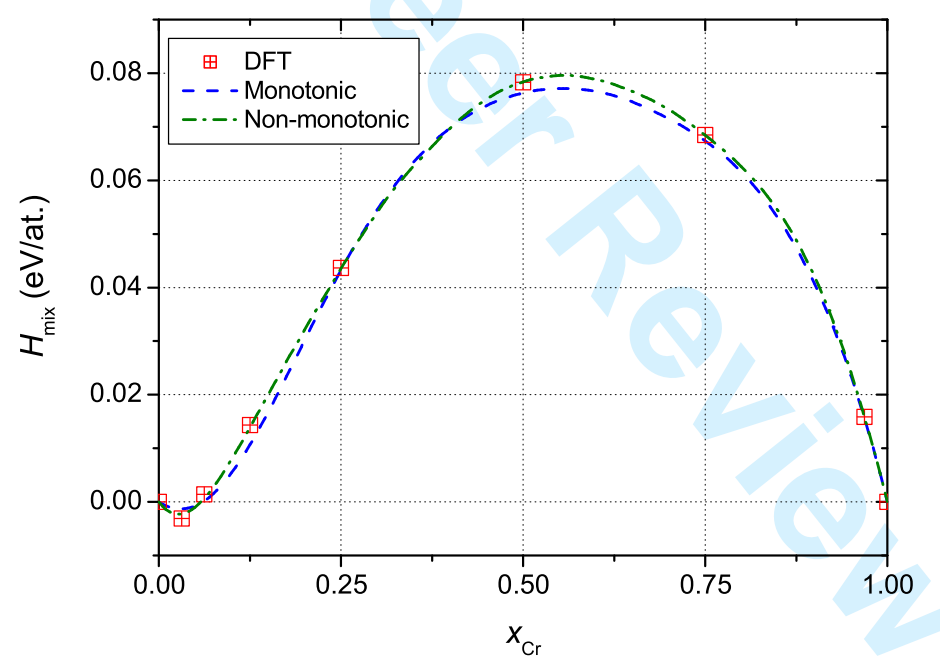

Figure 3. Mixing enthalpy for the two fitted 2BM potentials.

Fe-Cr $[5,16]$. In both fits the potential developed by Ackland et al. [17] and Olsson et al. [10] were taken for pure $\mathrm{Fe}$ and $\mathrm{Cr}$, respectively. This choice is the same as for the CDM by Caro et al. [11] and the 2BM by Olsson et al. [10].

The first potential (Potential 1) was built using a monotonically decreasing sdensity while the second (Potential 2) using a non-monotonic one, complying with equation (32). For both fits the same target mixing enthalpy and lattice parameter curve $a(x)$ were chosen; also the cut-off range was set to $5 \mathrm{nn}$ and the maximum s-density normalised to one. The mixing enthalpy resulting from the fits is shown in figure 3 and the resulting lattice parameter vs. concentration in figure 4 . The fitting parameters used in both fits are summarized in table 4 . From figure 3 it is clear that both potentials provide a very close fit to the target mixing enthalpy, especially Potential 2. The main difference among the two stems from the lattice parameter fit, 


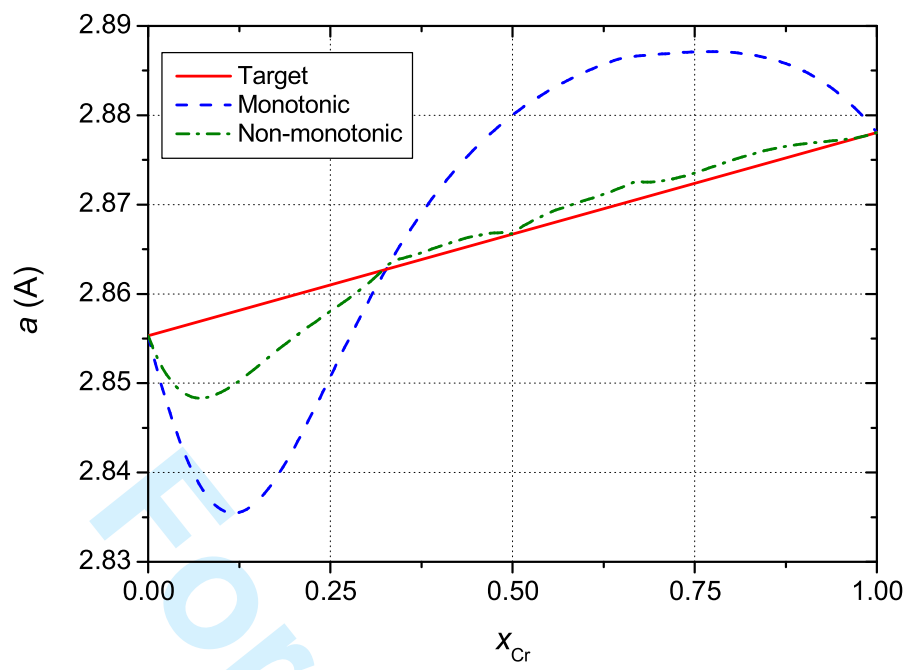

Figure 4. Id. figure 3 for the lattice parameter.

Table 1. Fitting parameters for both 2BM potentials.

\begin{tabular}{lll}
\hline Parameter & Potential 1 & Potential 2 \\
\hline$C_{0}$ & 0.529711608 & 0.0626506749 \\
$\alpha$ & 0.3 & - \\
$r_{0}$ & - & 3.0 \\
$\alpha_{1}$ & - & 2.0 \\
$\alpha_{2}$ & - & 0.97 \\
$v_{1}\left(r_{1}\right)$ & $0.0677704294(4.5)$ & $0.0905268178(4.5)$ \\
$v_{2}\left(r_{2}\right)$ & $-0.000299277931(3.733333$ & $-0.200654969(4.04)$ \\
$v_{3}\left(r_{3}\right)$ & $-1.82995956(2.96666667)$ & $0.780642508(3.58)$ \\
$v_{4}\left(r_{4}\right)$ & $-1.0(2.2)$ & $-2.53584652(3.12)$ \\
$v_{5}\left(r_{5}\right)$ & - & $0.715772831(2.66)$ \\
$v_{6}\left(r_{6}\right)$ & - & $-1.0(2.2)$ \\
$f_{1}^{\mathrm{Fe}}\left(\rho_{1}\right)$ & $0.70581525(0.0)$ & $2.95849728(0.0)$ \\
$f_{2}^{\mathrm{Fe}}\left(\rho_{2}\right)$ & $-1.01952346(0.333333333)$ & $-2.3715309(0.166666667)$ \\
$f_{3}^{\mathrm{Fe}}\left(\rho_{3}\right)$ & - & $-14.7655969(0.333333333)$ \\
$f_{4}^{\mathrm{Fe}}\left(\rho_{4}\right)$ & - & $40.5045783(0.5)$ \\
$f_{5}^{\mathrm{Fe}}\left(\rho_{5}\right)$ & - & $-55.7961184(0.666666667)$ \\
$f_{1}^{\mathrm{Cr}}\left(\rho_{1}\right)$ & $0.961221496(0.0)$ & $1.7898922(0.0)$ \\
$f_{2}^{\mathrm{Cr}}\left(\rho_{2}\right)$ & $-5.36653496(0.333333333)$ & $0.677998331(0.166666667)$ \\
$f_{3}^{\mathrm{Cr}}\left(\rho_{3}\right)$ & - & $-16.6914228(0.333333333)$ \\
$f_{4}^{\mathrm{Cr}}\left(\rho_{4}\right)$ & - & $34.5481014(0.5)$ \\
$f_{5}^{\mathrm{Cr}}\left(\rho_{5}\right)$ & - & $-74.3156461(0.666666667)$ \\
\hline
\end{tabular}

where Potential 2 clearly outperforms Potential 1; this is due to the non-monotonic s-density choice, which greatly simplifies the zero pressure condition, although with the above-mentioned shortcoming of sign-changing forces.

\section{Summary and conclusions}

A detailed analysis of the EAM and FS formalisms has shown the limitations of these models to fit concentration dependent properties of alloys. It has been shown that both the $2 \mathrm{BM}$ and $\mathrm{CDM}$ are equally capable of describing alloy properties that depend on concentration in a complex way. An illustration of this equivalence has been given, by deriving a 2BM potential out of a CDM one for the $\mathrm{Fe}-\mathrm{Cr}$ model alloy. A fitting algorithm for 2BM potentials has been proposed, capable of very 
closely fitting any mixing enthalpy shape. The algorithm has been applied to the Fe-Cr system and two potentials, that match the DFT calculated mixing enthalpy, were derived. It should be mentioned, however, that the potentials given here have only a demonstrative purpose and do not claim any generality for the study of other properties of interest for the Fe-Cr system, different from the mixing enthalpy.

\section{Acknowledgements}

This research has received partial funding from the European Atomic Energy Community's 7th Framework Programme (FP7/2007-2011), under grant agreement number 212175 (GetMat project). The work was also partially sponsored by the belgo-argentine MINCYT-FWO bilateral cooperation agreement, Project FW/07/EXII/002. RCP acknowledges support from CONICET-PICT 5062.

\section{References}

[1] B. Hennion, J. Phys. F 13 (1983) p.2351.

[2] P. Olsson, I.A. Abrikosov and J. Wallenius, J. Nucl. Mater. 321 (2003)p.84.

[3] A.A. Mirzoev, M.M. Yalalov and D.A. Mirzaev, Phys. Met. and Metall. 97 (2004) p.336.

[4] P. Olsson, I.A. Abrikosov and J. Wallenius, Phys. Rev. B 73 (2006) p.104416.

[5] P. Olsson, C. Domain and J. Wallenius, Phys. Rev. B 75 (2007) p.014110.

[6] M.S. Daw and M.I. Baskes, Phys. Rev. B 29 (1984) p.6443.

[7] M.W. Finnis and J.E. Sinclair, Phil. Mag. A 50 (1984) p.45.

[8] I. Mirebeau, M. Hennion and G. Parette, Phys. Rev. Lett. 53 (1984) p.687.

[9] N.P. Filippova, V.A. Shabashov and A.L. Nikolaev, The Physics of Metals and Metallography 90 (2000) p.145.

[10] P. Olsson, J. Wallenius, C. Domain, K. Nordlund and L. Malerba, Phys. Rev. B 72 (2005) p.214119.

[11] A. Caro, D.A. Crowson and M. Caro, Phys. Rev. Lett. 95 (2005) p.75702.

[12] G.J. Ackland and S.K. Reed, Phys. Rev. B 67 (2003) p.174108.

[13] O. Redlich and A.T. Kister, Indust. Eng. Chem. 40 (1948) p.345.

[14] N. Saunders and A.P. Miodownik, CALPHAD (Calculation of Phase Diagrams): A Comprehansive Guide, Pergamon, Oxford, 1998.

[15] G.J. Ackland and V. Vitek, Phys. Rev. B 41 (1990) p.10324.

[16] G. Bonny, R.C. Pasianot, L. Malerba, A. Caro, P. Olsson and M.Yu. Lavrentiev, Numerical prediction of thermodynamic properties of $\mathrm{Fe}$-Cr alloys using semi-empirical cohesive models: where do we stand?, accepted in J. Nucl. Mater. (2008).

[17] G.J. Ackland, M.I. Mendelev, D.J. Srolovitz, S. Han and A.V. Barashev, J. Phys: Condens. Matter 16 (2004) p.1.

\section{Appendix A. Site energy within CDM}

The concentration parameter $x_{\mathrm{AB}}$ of the CDM model is defined as,

$$
x_{\mathrm{AB}}^{i j}=\frac{1}{2}\left(\frac{\rho_{i}^{\mathrm{B}}}{\rho_{i}}+\frac{\rho_{j}^{\mathrm{B}}}{\rho_{j}}\right),
$$

for an A-B pair where $\mathrm{A}$ is at site $i$ and $\mathrm{B}$ at site $j$, with the obvious definition for the partial electron densities

$$
\rho_{i}^{\mathrm{B}}=\sum_{\nu \in i} n_{\mathrm{B}}^{\nu} \varphi_{\mathrm{B}}^{\nu}
$$

In the denominators of equation (A1) we may disregard the non-essential fact that the electron density functions are generally different, namely, we assume $\varphi_{\mathrm{A}}(r)=$ $\varphi_{\mathrm{B}}(r)=\varphi(r)$. This choice makes the total density homogeneous throughout the lattice and also the average of the partial density for the B species, namely, $\forall i \rho_{i}=\rho$ 
and ${\overline{\rho_{i}}}^{\mathrm{B}}=\rho^{\mathrm{B}}$. This observation however does not destroy the statistical correlation between the two terms in equation (A1); there, $\bar{\rho}_{i}^{\mathrm{B}}$ will be above average, whereas ${\overline{\rho_{j}}}^{\mathrm{B}}$ below. It is easy to see that

$$
\begin{aligned}
& {\overline{\rho_{i}}}^{\mathrm{B}}=\rho^{\mathrm{B}}+x_{\mathrm{A}} \varphi\left(r_{i j}\right) \\
& {\overline{\rho_{j}}}^{\mathrm{B}}=\rho^{\mathrm{B}}-x_{\mathrm{B}} \varphi\left(r_{i j}\right)
\end{aligned}
$$

with $\rho^{\mathrm{B}}=x_{\mathrm{B}} \sum_{\mu} Z_{\mu} \varphi\left(R_{\mu}\right)=x_{\mathrm{B}} \rho$. The application of this result to equation (A1) leads to

$$
\bar{x}_{\mathrm{AB}}^{\nu}=x_{\mathrm{B}}+\left(x_{\mathrm{A}}-x_{\mathrm{B}}\right) \frac{\varphi\left(r_{\nu}\right)}{2 \rho} .
$$

The variance of equation (A1) is more difficult to give in a closed expression; we may view it with the help of the following identity expressing the variance for the addition of two variables $X$ and $Y$,

$$
\overline{(X+Y)^{2}}-\overline{(X+Y)}^{2}=\left(\overline{X^{2}}-\bar{X}^{2}\right)+\left(\overline{Y^{2}}-\bar{Y}^{2}\right)+2(\overline{X Y}-\bar{X} \bar{Y}) .
$$

The first two terms of the r.h.s. above are clearly equal when applied to equation (A1), this common value is named $\sigma_{\nu}^{2}$. The last term, because of the sites shared by the two nodes $i$ and $j$ of the pair, varies between $2 \sigma_{\nu}^{2}$ for the highly correlated case (close pairs) and 0 for the uncorrelated one (well separated pairs). Using now the variance formula for the binomial distribution one obtains,

$$
\sigma_{\nu}^{2}=x_{\mathrm{A}} x_{\mathrm{B}} \frac{\sum_{\mu} Z_{\mu} \varphi\left(r_{\mu}\right)^{2}-\varphi\left(r_{\nu}\right)^{2}}{4 \rho^{2}}
$$

that leads to the variance of the concentration parameter,

$$
\overline{\left(x_{A B}^{\nu}-\bar{x}_{A B}^{\nu}\right)^{2}}=C_{\nu} \sigma_{\nu}^{2},
$$

where $C_{\nu}$ is a constant that varies between 2 and 4 . With expressions (A5), (A8), and (13) the average site energy within the CDM formalism is completely determined as a function of the global concentration.

Appendix B. Equivalence in alloy energetics between 2BM and CDM

The 2BM and CDM formalisms are here compared. The total energy within CDM can be written as,

$$
E_{\mathrm{CDM}}=E_{\mathrm{EAM}}+\frac{1}{2} \sum_{i, j}\left(h\left(x_{i j}\right)-1\right) V_{\mathrm{AB}}\left(r_{i j}\right)\left(1-\delta_{t_{i} t_{j}}\right),
$$

where $t_{i}$ is the atom-type occupying site $i$ and $\delta$ the Kronecker delta. Within 2BM the total energy is in turn,

$$
E_{2 \mathrm{BM}}=E_{\mathrm{EAM}}+\sum_{i} F_{t_{i}}^{\mathrm{s}} .
$$


The latter term of the above equation can easily be recasted as,

$$
\begin{aligned}
\sum_{i} F_{t_{i}}^{\mathrm{s}} & =\sum_{i} \frac{F_{t_{i}}^{\mathrm{s}}}{\rho_{i}^{\mathrm{s}}} \rho_{i}^{\mathrm{s}} \\
& =\sum_{i, j(i \neq j)} \frac{F_{t_{i}}^{\mathrm{s}}}{\rho_{i}^{\mathrm{s}}} \varphi_{t_{i} t_{j}}^{\mathrm{s}}\left(r_{i j}\right) \\
& =\frac{1}{2} \sum_{i, j(i \neq j)}\left(\frac{F_{t_{i}}^{\mathrm{s}}}{\rho_{i}^{\mathrm{s}}}+\frac{F_{t_{j}}^{\mathrm{s}}}{\rho_{j}^{\mathrm{s}}}\right) \varphi_{t_{i} t_{j}}^{\mathrm{s}}\left(r_{i j}\right) \\
& =\frac{1}{2} \sum_{i, j}\left(\frac{F_{t_{i}}^{\mathrm{s}}}{\rho_{i}^{\mathrm{s}}}+\frac{F_{t_{j}}^{\mathrm{s}}}{\rho_{j}^{\mathrm{s}}}\right) \varphi_{\mathrm{AB}}\left(r_{i j}\right)\left(1-\delta_{t_{i} t_{j}}\right) .
\end{aligned}
$$

Identifying equations (B1) and (B2) one finds the relationship between both formalisms,

$$
\left(\frac{F_{t_{i}}^{\mathrm{s}}}{\rho_{i}^{\mathrm{s}}}+\frac{F_{t_{j}}^{\mathrm{s}}}{\rho_{j}^{\mathrm{s}}}\right) \varphi_{A B}^{\mathrm{s}}\left(r_{i j}\right)=\left(h\left(x_{i j}\right)-1\right) V_{\mathrm{AB}}\left(r_{i j}\right) .
$$

The left factors in both sides of equation (B4) are functions of a variable depending on the local concentration, while the right factor is a function depending on the distance between two atoms. Heuristically, one can further identify,

$$
\begin{aligned}
\frac{F_{\mathrm{A}}^{\mathrm{s}}\left[\rho_{\mathrm{A}}^{\mathrm{s}}(x)\right]}{\rho_{\mathrm{A}}^{\mathrm{s}}(x)}+\frac{F_{\mathrm{B}}^{\mathrm{s}}\left[\rho_{\mathrm{B}}^{\mathrm{s}}(x)\right]}{\rho_{\mathrm{B}}^{\mathrm{s}}(x)} & =1-h(x) \\
\varphi_{\mathrm{AB}}^{\mathrm{s}}(r) & =-V_{\mathrm{AB}}(r) .
\end{aligned}
$$

Formally, there is no one-to-one relationship between $\rho^{\mathrm{s}}$ and $x_{\mathrm{AB}}$, since both quantities depend on the local concentration, which can be different from the global concentration $x$. Practically, equations (B5) and (B6) suggest that choosing a nonmonotonic s-density within the $2 \mathrm{BM}$ formalism is equivalent to the development of a CDM potential. 


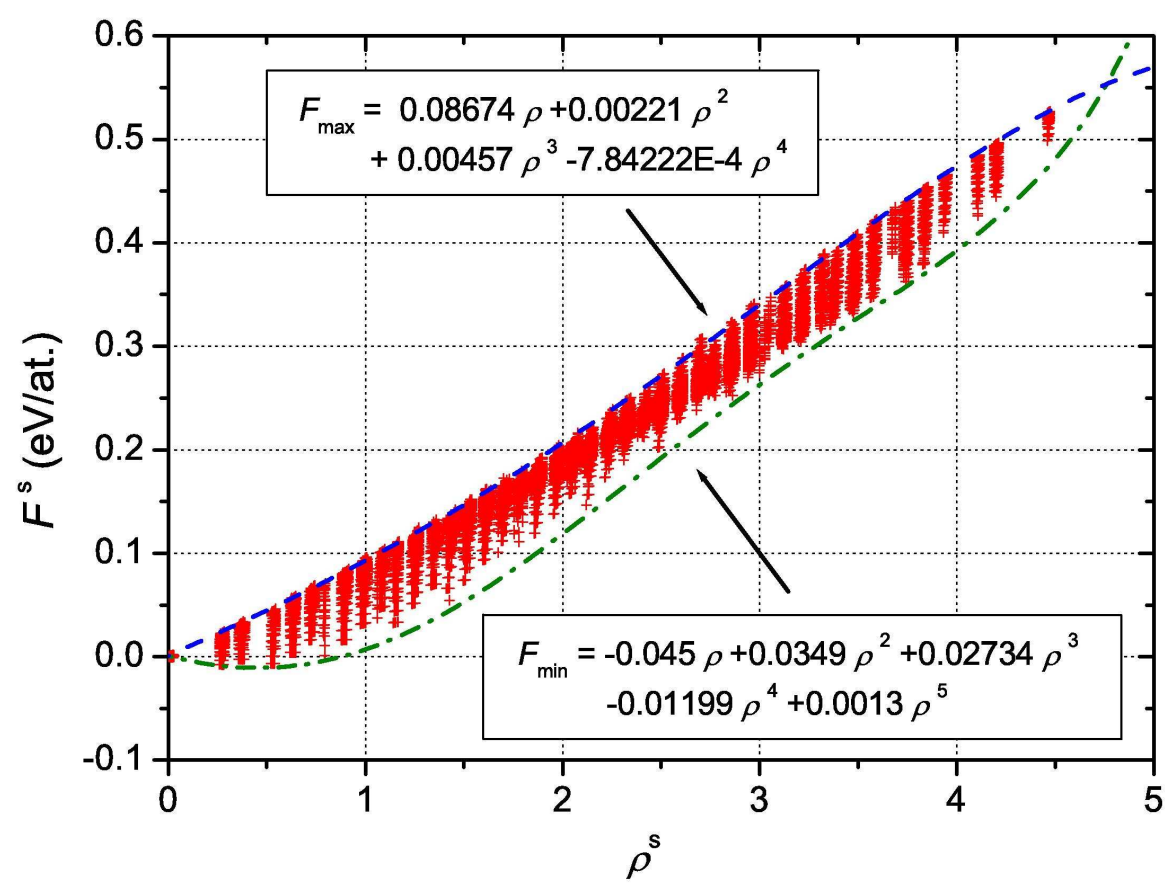

Possible s-embedding functions for Fe as a function of s-density. $104 \times 81 \mathrm{~mm}(600 \times 600 \mathrm{DPI})$ 


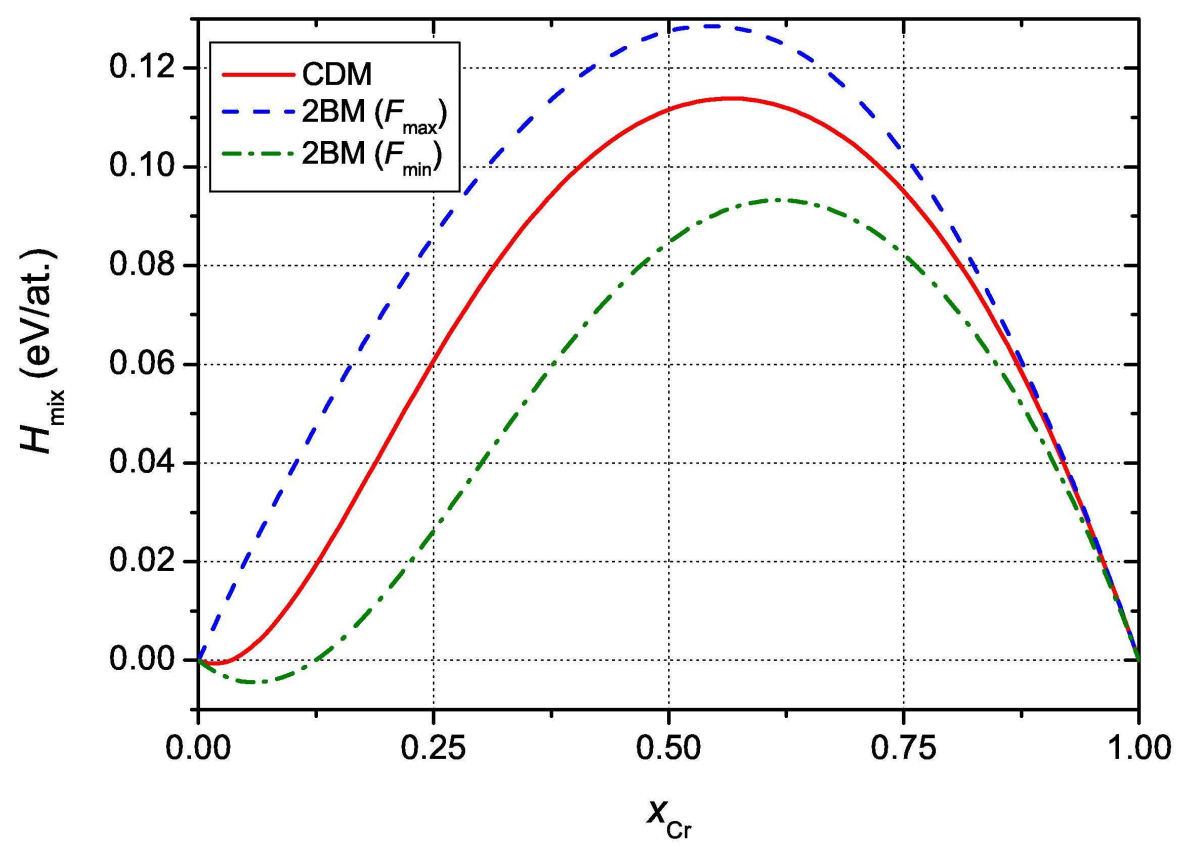

Mixing enthalpy for the $\mathrm{CDM}$ and therefrom derived 2BM potentials. $109 \times 80 \mathrm{~mm}(600 \times 600 \mathrm{DPI})$ 


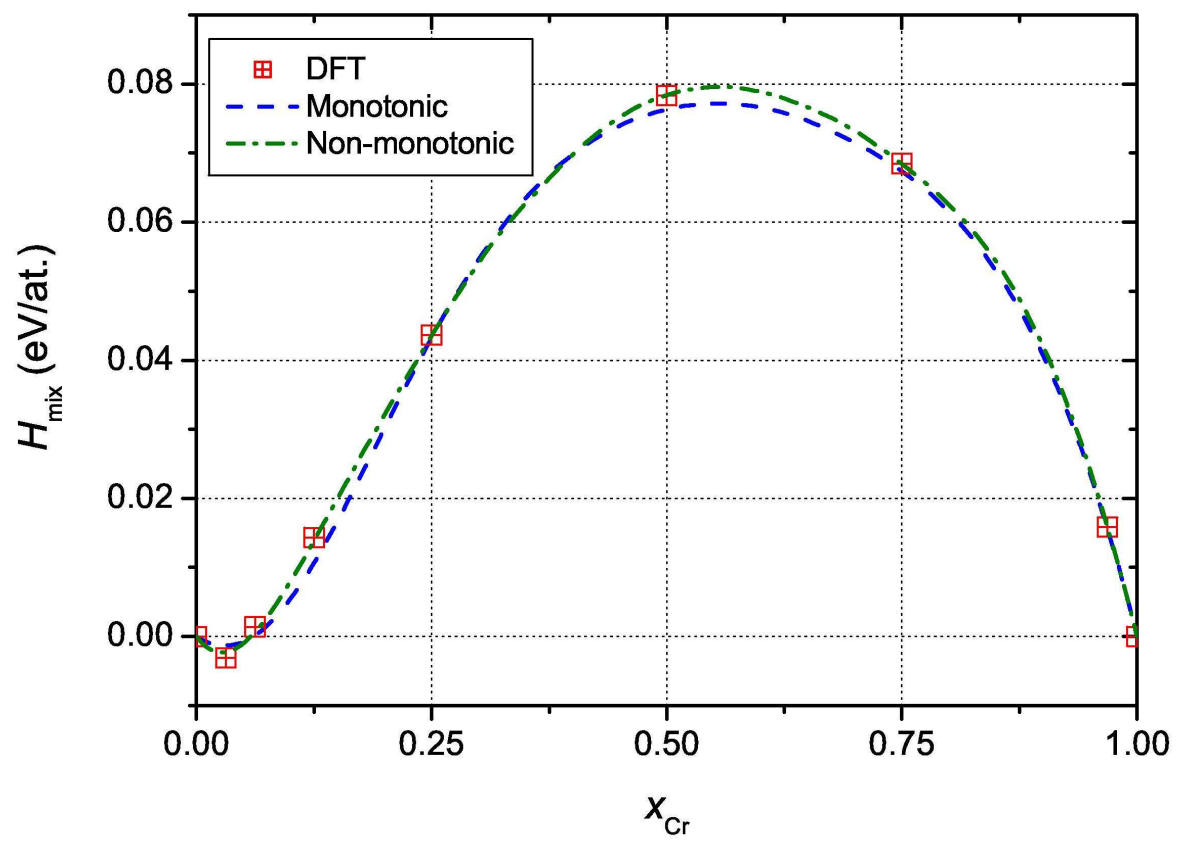

Mixing enthalpy for the two fitted 2BM potentials. $109 \times 80 \mathrm{~mm}(600 \times 600 \mathrm{DPI})$ 


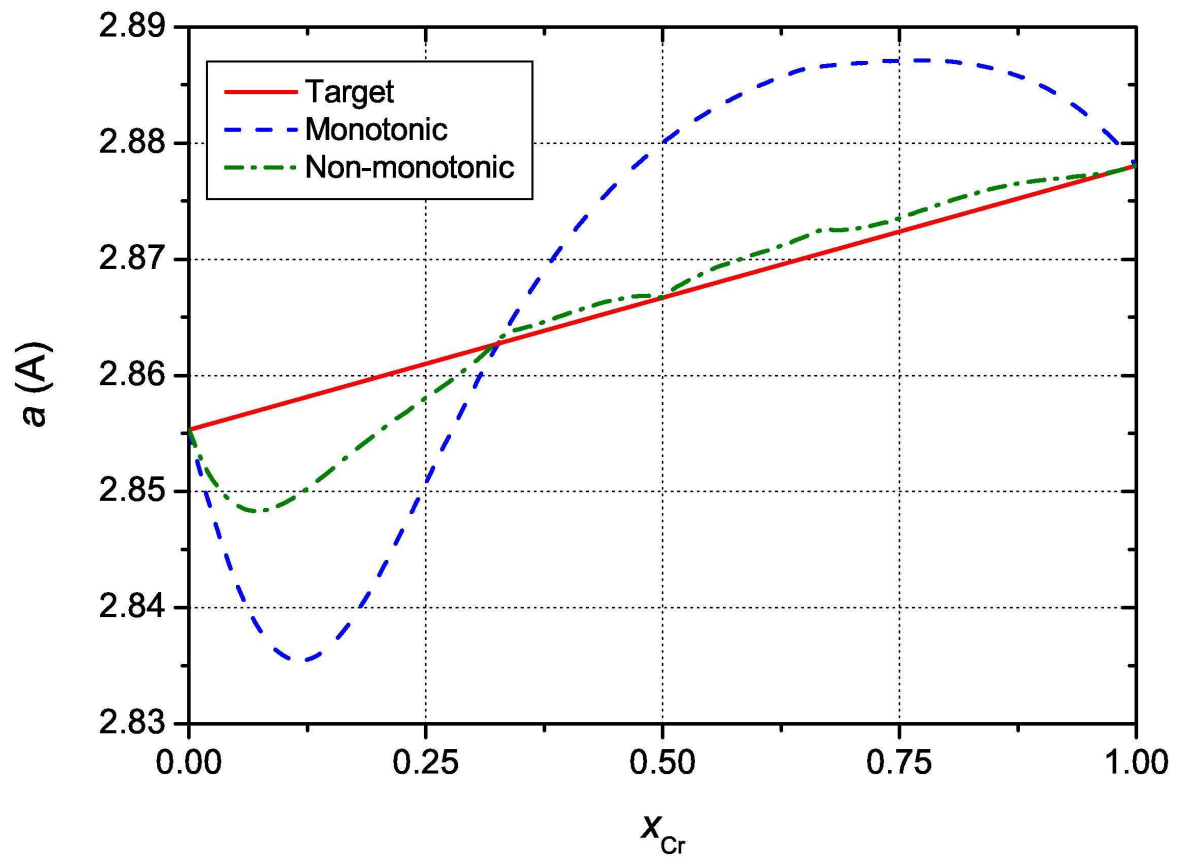

Id. figure 3 for the lattice parameter. $108 \times 82 \mathrm{~mm}(600 \times 600 \mathrm{DPI})$ 